\title{
Recognition of flavivirus species on the base of coding genome sequences
}

\author{
Maria Chaley \\ Institute of Mathematical Problems of Biology RAS - \\ Branch of Keldysh Institute of Applied Mathematics RAS \\ Pushchino, Russia \\ chaley@phystech.edu
}

\author{
Vladimir Kutyrkin \\ Department of Comp. Mathematics and Math. Physics \\ Moscow State Technical University n.a. N.E. Bauman \\ Moscow, Russia \\ vkutyrkin@yandex.ru
}

\begin{abstract}
Computational method for recognition the flavivirus species, including their subtypes, is proposed, that based on known sequence of viral genome. The method uses frequency characteristics of amino acid codons in the whole coding sequences of polyproteins. High reliability of this method is supported by correct recognizing of the 15 groups from different species and subtypes of flavivirus genomes.
\end{abstract}

Keywords - flavivirus genome; latent profile triplet periodicity; flavivirus species recognition

\section{Introduction}

Flaviviruses belong to genus of arboviruses from Flaviridae family and they are transmitted through the mosquito and tick stings. Flaviviruses invade nervous system, inducing heavy diseases of human along with domestic and wild animals and birds [1-5]. Among them the most known diseases are tickborne encephalitis, dengue fever, yellow fever, West Nile fever. To identify flavivirus species or subtype the reverse transcription and polymerase chain reaction with further cDNA sequencing of viral genome are applied along with traditional enzyme-linked immunosorbent assay (ELISA). Nucleotide sequence obtained in the result is compared with other known viral genome sequences in the GenBank.

Flavivirus genome is represented by single-stranded RNA of positive polarity, having length about 11 thousand nucleotides. Sequentially arranged with each other and translated in the same reading frame, the genes of viral proteins form a whole coding sequence (CDS) of polyprotein.

In present work the 15 groups of flavivirus polyprotein CDSs with total number of the representatives equal to 7060 were considered. Whole coding sequences of ten flavivirus species such as Bagaza virus, Japanese encephalitis virus, Murray Valley encephalitis virus, Powassan virus, Saint Louis encephalitis virus, Duck Tembusu virus, Tick-borne encephalitis virus, Usutu virus, the viruses of West Nile and Yellow fevers were analyzed. Besides, the four groups of polyproteins' CDSs were separately considered for the subtypes of Dengue fever virus (Dengue serotype 1, Dengue serotype 2, Dengue serotype 3, Dengue serotype 4), as well as Kunjin virus which is now considered as Australian subtype of West Nile fever virus was distinguished in a special group. In all analyzed CDSs of flavivirus proteins latent triplet periodicity was recognized, that is referred to earlier introduced new type of profile periodicity [6]. As more general model, explaining existence of such the latent periodicity, so called the SHOC-model has been proposed [7]. This model is unambiguously characterized by random codon which is a random value with the meanings in triplets of the genetic code. Consequently, in frame of the SHOC-model for coding DNA sequence, where the latent triplet periodicity is observed, an estimate for random codon's probability distribution may be obtained as a set (vector) of frequencies of the genetic codons occurred in this sequence. So, such the DNA sequences may be classified according to the types of the random codon distributions.

Thereby, analyzed 15 groups of the CDSs of flavivirus polyproteins induce a set of corresponding sampled probability distributions, forming a set of the points in the 64-dimentional space. Clusterization problem for such the points' set, where each distinct cluster is characterized by only one of the species or subtypes of the flavivirus considered, is addressed in the work. Algorithm proposed in the work is based on sampled probability distribution of the codons and it allows unambiguously assigning polyprotein CDS to a cluster of corresponding flavivirus species or subtype.

\section{Materials and Methods}

In the present work flavivirus genomes were selected from the GenBank release 231.0, dated April 15 2019. It should be noted that the introns in flavivirus genomes are practically absent, so flavivirus genome CDS is represented by uninterrupted coding sequence of polyprotein. Table I presents qualitative $(\mathbf{j})$ and quantitative $\left(\mathbf{N}_{\mathbf{j}}\right)$ content of the 15 groups of flavivirus polyprotein CDSs analyzed.

TABLE I

\begin{tabular}{|c|l|c|}
\hline $\mathrm{j}$ & \multicolumn{1}{|c|}{ Species and subtypes of flaviviruses } & $\mathrm{N}_{\mathrm{j}}$ \\
\hline 1 & Bagaza virus & 18 \\
\hline 2 & Dengue serotype 1 virus & 1895 \\
\hline 3 & Dengue serotype 2 virus & 1412 \\
\hline 4 & Dengue serotype 3 virus & 922 \\
\hline 5 & Dengue serotype 4 virus & 190 \\
\hline 6 & Japanese encephalitis virus & 300 \\
\hline 7 & Kunjin virus & 43 \\
\hline 8 & Murray Valley encephalitis virus & 14 \\
\hline 9 & Powassan virus & 21 \\
\hline 10 & Saint Louis encephalitis virus & 38 \\
\hline 11 & Duck Tembusu virus & 110 \\
\hline 12 & Tick-borne encephalitis virus & 175 \\
\hline 13 & Usutu virus & 147 \\
\hline 14 & West Nile virus & 1652 \\
\hline 15 & Yellow fever virus & 123 \\
\hline
\end{tabular}


The mean value of frequency vector of the codons of the genetic code was calculated over all the genomes for each group. Such a vector unambiguously characterizes corresponding group, i. e. flavivirus species or subtype (serotype).

Question of identity with one or another group for flavivirus genome analyzed was solved by the following way. Original statistics was proposed in the work that calculates a distance of frequency vector for individual flavivirus genome from the mean values of frequency vectors for all other groups. Flavivirus genome analyzed was attributed to a group that showed minimum of distance between such the vectors. To prove a correctness of group choice the following additional procedure was used. For flavivirus genomes in each group a maximal distance of all corresponding codon frequency vectors from the mean value of codon frequency vector in the group was calculated. Choice of identity group was confirmed, if minimal distance found was no more such the maximal distance in the group.

\section{Results}

Method proposed in the work allows determining flavivirus species, if coding sequence of its polyprotein is known. This method was tested in recognition of 10 species and five subtypes of the flaviviruses with the genomes significantly represented in the GenBank. It was shown [8] that for 10 species and two subtypes the method provides $100 \%$ reliability. Mistakes in flavivirus species recognition amount to no more than $0.5 \%$. In particular, 23 genomes among 7060 flavivirus genomes analyzed were not recognized and it was only two cases of wrong species determination.

\section{Conclusion}

New, effective and rapid method was proposed in the work to identify the species of flaviviruses, giving rise to heavy diseases of human, animals and birds, after viral genomes have been sequenced.
In general, application of the method proposed is not restricted by identifying RNA viruses only, particularly, flaviviruses. The method may be used for proving virus species from other genus and families, independently of RNA or DNA viruses are analyzed.

Method proposed in the work significantly simplifies a procedure of virus identification that is based on sequence of its genome, as no additional knowledge about conservative fragments of viral genome is needed, because alignment with other viral genomes from international databases is not carried out.

\section{REFERENCES}

[1] J. Fernández-Pinero, I. Davidson, M. Elizalde et al., "Bagaza virus and Israel turkey meningoencephalomyelitis virus are a single virus species," J. Gen. Virol., vol. 95, pp. 883-887, 2014.

[2] W. Zhang, S. Chen, S. Mahalingam, et al., "An updated review of avianorigin Tembusu virus: a newly emerging avian Flavivirus,". J. Gen. Virol., vol. 98, pp. 2413-2420, 2017.

[3] E. Benzarti, A. Linden, D. Desmecht, et al., "Mosquito-borne epornitic flaviviruses: an update and review," J. Gen. Virol., vol. 100, pp. 119$132,2019$.

[4] A. Diaz, L. Coffey, N. Burkett-Cadena, et al., "Reemergence of St. Louis encephalitis virus in the Americas. Emerging Infectious Diseases," vol. 24., pp. 2150-2157, 2018.

[5] M. Clé, C. Beck, S. Salinas, et al., "Usutu virus: A new threat?", Epidemiol. Infect., vol. 147. Article No. e232, 2019.

[6] M. Chaley, V. Kutyrkin, "Spectral-statistical approach for revealing latent regular structures in DNA sequences," in Data Mining Techniques for the Life Sciences, O. Carugo, F. Eisenhaber, Eds. New York: Springer Science+Business Media, 2016, pp. 315-340.

[7] M. Chaley, V. Kutyrkin, "Stochastic model of homogeneous coding and latent periodicity in DNA sequences," J. Theor. Biol., vol. 390. pp. 106116, 2016.

[8] M. Chaley, Zh. Tyulko, V. Kutyrkin, "Flavivirus species recognition based on the polyprotein coding sequences," Math. Biol. Bioinf., vol. 14, pp. 533-542, 2019. (In Russian) 